\title{
Aberrant ceRNA activity drives lung cancer
}

\author{
Cell Research (2014) 24:259-260. doi:10.1038/cr.2014.21; published online 14 February 2014
}

\begin{abstract}
In a recent issue of Nature, Kumar et al. demonstrate that the oncogenic potential of the $\mathrm{Hmga} 2$ gene is largely due to the ability of its transcript to operate as a competing endogenous RNA in a protein coding-independent manner. The Hmga2 mRNA decoys the let-7 microRNA family to regulate Tgfbr3 expression and enhance TGF- $\beta$ signaling, thereby promoting lung cancer progression.
\end{abstract}

MicroRNAs are a large class of small non-coding RNAs which regulate gene expression by binding to microRNA response elements (MREs) on target transcripts. It has become increasingly clear in recent years that transcripts which contain MREs for shared microRNAs can co-regulate each other by titrating microRNA availability, thus acting as natural microRNA sponges or competing endogenous RNAs (ceRNAs) [1, 2]. Although ceRNA activity has been attributed to both protein-coding and non-coding RNAs such as small noncoding RNAs, long non-coding RNAs, pseudogenes and circular RNAs in diverse species, little is known about the precise molecular conditions needed for optimal ceRNA crosstalk.

A recent study by Kumar et al. has led to important insights into this recently described post-transcriptional regulatory dimension [3]. The authors focused on Hmga2, a non-histone chromosomal high-mobility group protein which contributes to lung cancer progression and metastasis. The Hmga2 $3^{\prime}$ UTR contains seven conserved MREs for the let-7 family of microRNAs [4], which has previously been demonstrated to constrain lung cancer development [5]. Kumar et al. generated a series of
Hmga2 expression constructs (wildtype Hmga 2, WT; Hmga2 with all seven let- 7 binding sites mutated, $\mathrm{m}$ 7; Hmga2 with the single in-frame start codon mutated, ATG WT; and Hmga2 with both the start codon and let-7 binding sites mutated, ATG $\mathrm{m} 7$ ). This allelic series enabled them to disentangle the contribution of $\mathrm{Hmga2}$ 's function as a let-7 ceRNA from its protein-coding function in lung cancer development.

Kumar et al. found that both WT and ATG WT constructs dramatically promoted anchorage-independent growth of lung cancer cell lines in vitro, rescued tumor formation in an autochthonous lung cancer model in vivo, and reduced survival of transplanted mice. A more modest effect was observed with the $\mathrm{m} 7$ construct and no growth was observed with the double mutant ATG m7 construct. Critically, the in vitro effects were rescued by exogenous expression of let-7. These results indicate that the interaction of the Hmga2 transcript with let-7 is critical for the oncogenic function while the Hmga2 protein is largely dispensable for lung cancer progression, suggesting a ceRNA function for the Hmga 2 transcript.

To examine potential targets of Hmga2's ceRNA activity, the authors predicted let-7 targets among mRNAs that are differentially expressed in Hmga2-expressing vs non-expressing lung cancer cells. This approach identified six ceRNA targets that are regulated by Hmga 2 in a let-7-dependent manner. Subsequent analyses validate the TGF- $\beta$ co-receptor Tgfbr3 as a bona fide target of Hmga2 ceRNA (Figure 1A), and establish Tgfbr 3 and TGF- $\beta$ signaling to be critical for the oncogenic effect of Hmga2. Importantly, HMGA2 and $T G F B R 3$ expression is reciprocally positively correlated in human lung cancer, indicating that the oncogenic ceRNA function of HMGA2 is conserved across species.

Kumar et al. performed several important experiments that further validated ceRNA crosstalk between Hmga2 and $T g f b r 3$ and addressed a number of key open questions about such crosstalk. First, the authors demonstrated that $T g f b r 3$ recruitment to the Ago2-based microRNA repression complex was reduced in both Hmga2 WT and ATG WT cells by RNA immunoprecipitation. Intriguingly, the authors reported that Hmga2's ceRNA activity does not result in changes in the levels of let-7 isoforms or their association with Ago2, suggesting that Hmga2 was able to block the association of $T g f b r 3$ with Ago 2 via its function as a let-7 ceRNA. As previous studies have shown that microRNA decoying can direct the degradation and thus decrease the abundance of decoyed microRNAs [6], this observation suggests that ceRNA regulation may have varying effects on targeted microRNAs which may be sequence-specific and context-dependent.

Moreover, RNA-seq analyses demonstrated that Hmga2 was one of the most highly expressed predicted let-7 target transcripts, Hmga2 and Tgfbr3 transcripts were expressed at similar levels, and total expression levels of let7 family members was within an order of magnitude of Hmga2 and Tgfbr3. These observations are consistent with previous reports suggesting that optimal ceRNA crosstalk would occur when the abundance of microRNA and ceRNA 


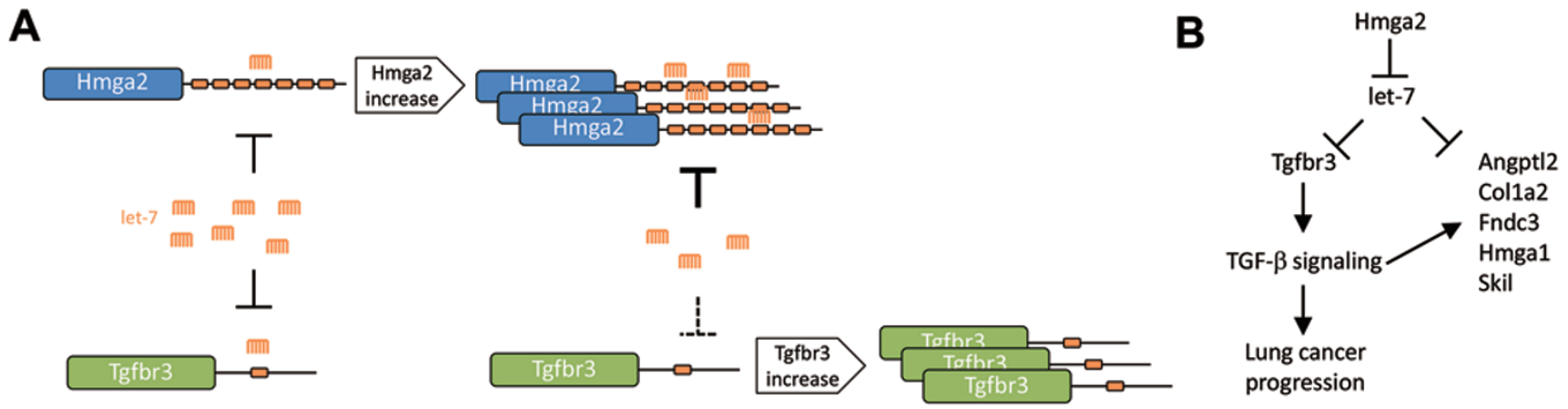

Figure 1 (A) Hmga2 acts as a natural microRNA sponge for the let-7 family, which also targets Tgfbr3. An increase in Hmga2 transcript levels and hence ceRNA activity results in a concomitant increase in Tgfbr3 levels. (B) Hmga2 also regulates the expression of five other target genes via ceRNA crosstalk as well as through Tgfbr3/TGF- $\beta$-mediated transcriptional control.

transcripts were near equimolarity [7]. Hmga2 shares let-7 sites with numerous other transcripts besides $T g f b r 3$ and, considering simple stoichiometry, the effect of reduced let-7 availability in response to Hmga2 overexpression should be "diluted" by all let-7 targets. Indeed, using Sylamer analysis, Kumar et al. report an enrichment of let-7 site-containing transcripts upon Hmga 2 overexpression, suggesting a more global effect on let-7 targets. Intriguingly, however, only six differentially expressed let- 7 targets were identified as Hmga 2 ceRNA targets. These data could indicate a marginal effect of Hmga2-mediated sequestration of let-7 on most let-7 targets, while a few let-7 targets are affected signficantly. Stoichiometry dictates that the overexpression of an abundant transcript such as $\mathrm{Hmga} 2$ has profound ceRNA effects on lowly expressed let-7 targets. However, five out of six identified Hmga 2 ceRNA targets (Tgfbr3, Angptl2, Fndc3, Hmgal, and Skil) are among the highly expressed let-7 targets, suggesting that additional factors such as subcellular localization may determine the effect of let-7 sponging. Indeed, another prominent target of the let-7 family, the potent oncogene K-Ras, was unaffected by Hmga 2 overexpression. Future work will provide further insight into the intricacies of regulation of ceRNA crosstalk. Additionally, recent reports have identified other bona fide
Hmga2-targeting microRNAs, including miR-33a and miR-154 [8,9]. The ability of Hmga 2 to sequester these and other additional microRNAs may confer further complexity to its function as a ceRNA and should be investigated in future studies.

This study also provided evidence for the co-evolution of ceRNA and transcriptional networks. Kumar et al. found that the five Hmga2 ceRNA targets besides $T g f b r 3$ are transcriptionally regulated by TGF- $\beta$ signaling. This suggests that Hmga2 regulates the expression of these transcripts in a feed-forward manner, first through ceRNA crosstalk, and second through transcriptional control via the Tgfbr3TGF- $\beta$ axis (Figure 1B). Many more of such integrated ceRNA and transcriptional networks likely remain to be found, and their identification would indicate that ceRNA crosstalk is not a mere coincidence but a sophisticated means to fine-tune biological networks.

In summary, this report by Kumar et al. provides convincing evidence for the provocative hypothesis that in specific contexts, the primary function of a protein-coding gene may act as a microRNA decoy. It is also the first study which provides mutational analyses of the relevant microRNA-binding sites to demonstrate the functional relevance of ceRNA activity in tumorigenesis in vivo. Furthermore, Kumar et al. demonstrate that even highly abundant microRNAs like let-7 can be effectively regulated by highly expressed ceRNAs with multiple MREs. As both Hmga2 and let-7 have been implicated in multiple cancers as well as developmental processes, further insights into the Hmga2-let7 ceRNA network will have important implications for human health and disease.

Yvonne Tay ${ }^{1}$, Florian A Karreth ${ }^{1}$, Pier Paolo Pandolfi ${ }^{1}$

\footnotetext{
${ }^{I}$ Cancer Research Institute, Beth Israel Deaconess Cancer Center, Department of Medicine and Pathology, Beth Israel Deaconess Medical Center, Harvard Medical School, Boston, MA 02215, USA

Correspondence: Pier Paolo Pandolfi

Tel: +1-617-735-2121; Fax: +1-617-735-2120

E-mail: ppandolf@bidmc.harvard.edu
}

\section{References}

1 Salmena L, Poliseno L, Tay Y, et al. Cell 2011; 146:353-358.

2 Tay Y, Rinn J, Pandolfi PP. Nature 2014; 505:344-352.

3 Kumar MS, Armenteros-Monterroso E, East $\mathrm{P}$, et al. Nature 2014; 505:212-217.

4 Mayr C, Hemann MT, Bartel DP. Science 2007; 315:1576-1579.

5 Kumar MS, Erkeland SJ, Pester RE, et al. Proc Natl Acad Sci USA 2008; 105:39033908.

6 Cazalla D, Yario T, Steitz JA. Science 2010; 328:1563-1566.

7 Ala U, Karreth FA, Bosia C, et al. Proc Natl Acad Sci USA 2013; 110:7154-7159.

8 Rice SJ, Lai SC, Wood LW, et al. J Biol Chem 2013; 288:16348-16360.

9 Zhu C, Li J, Cheng G, et al. Mol Cell Biochem 2013; 379:69-75. 